Thorax, 1978, 33, 167-174

\title{
Pulmonary noradrenergic innervation of rat and monkey: a comparative study
}

\author{
AL-WALID I. EL-BERMANI
}

From the Department of Anatomy, Tufts University School of Medicine, 136 Harrison Avenue, Boston, Massachusetts 02111, USA

El-Bermani, Al-Walid I. (1978). Thorax, 33, 167-174. Pulmonary noradrenergic innervation of rat and monkey: a comparative study. The noradrenergic innervation of rat and monkey lungs was studied using the fluorescence histochemical method for norepinephrine of Falck (1962). In both species the noradrenergic nerves enter the lung at the hilum in association with the bronchial arteries. Major differences were noted in the distribution and pattern of these nerves, the most important of which are as follows: (1) Noradrenergic nerves have terminal varicosities in all divisions of the rat bronchial artery but are varicose in only the medium and small bronchial arteries of the monkey. (2) Noradrenergic terminals (varicosities) are in direct association with the bronchial smooth muscle in the monkey, but in the rat most of the noradrenergic nerves pass through the smooth muscle layer without forming terminal varicosities. Smooth muscle noradrenergic innervation is seen only at bifurcation points. (3) In the monkey pulmonary artery, noradrenergic terminals are restricted to the adventitio-medial junction while they appear in the media of the rat pulmonary artery. (4) Noradrenergic terminals are present in all pulmonary vein divisions of the monkey whereas in the rat the large pulmonary veins lack noradrenergic nerves.

Both rat and monkey bronchial arteries and pulmonary veins were found to have adrenergic terminal varicosities deep in the medial layer. The rat pulmonary artery also has such medial terminations. In both species there is a preponderance of noradrenergic terminals in the vasa vasorum, and we think that this may be important in regulating the delivery of humoral substances to vascular walls.

It has been established that the autonomic nervous system contributes innervation to the lung in many vertebrate species (Larsell, 1921, 1922; von Hayek, 1960), and stimulation of the sympathetic nerves to the lung releases catecholamines into the venous effluent (Lockett, 1957). The innervation of blood vessels of the lung was identified histologically by Larsell (1921), Honjin (1956), and Fisher (1965). Most histochemical studies of blood vessel innervation have been on the systemic circulation. These studies have uniformly shown adrenergic nerves to be concentrated at the adventitio-medial junction (for example, Falck (1962); Fuxe and Sedvall (1965); Ehinger et al. (1967)). The few studies of pulmonary vascular nerves have also shown nerve terminals mainly in the adventitial layer (Altenähr, 1965; Zussman, 1966; Čech and Doležel, 1967; Fillenz, 1967).
Some authors have noted a dearth of vascular innervation, and controversy has arisen regarding the distribution of various types of nerves (Cech and Doležel, 1967; Cech, 1969). We have noted during histochemical studies of rat and monkey lung that, in some areas, there is dense adrenergic vascular innervation in the media, and we have therefore undertaken a systematic study of the pulmonary adrenergic nerves in these two species.

\section{Methods and material}

More than 50 adult Wistar rats weighing 150 $300 \mathrm{~g}$ and 15 adult Macaca rhesus monkeys were used for the present study. Lungs, judged to be free of disease at the time of surgery, were removed from anaesthetised animals, and $1-\mathrm{cm}$ cubes of lung tissue were immediately frozen on 
dry ice. Tissue was sectioned in the cryostat at $-20^{\circ} \mathrm{C}$, and $15-20 \mu \mathrm{m}$ sections were collected in aluminium trays over dry ice. Sections were then transferred to a Virtis freeze dryer at $-50^{\circ} \mathrm{C}$ and a vacuum of $25 \mu \mathrm{m}$ and freeze-dried for 12 hours. The freeze dryer was then slowly brought to room temperature, and the tissue was placed in a sealed container for exposure to standardised paraformaldehyde for one hour at $80^{\circ} \mathrm{C}$. Sections were mounted in paraffin oil and examined immediately by a fluorescence microscope with appropriate filters according to the method of Falck (1962).

\section{Results}

RAT

The majority of the noradrenergic nerves observed at the hilum of the rat lung are associated with the bronchial arteries. After the reaction with paraformaldehyde vapour, fluorescent fibres can be seen to surround the bronchial arteries in a dense network. They have specific orientation to both the longitudinal and the circumferential axis of

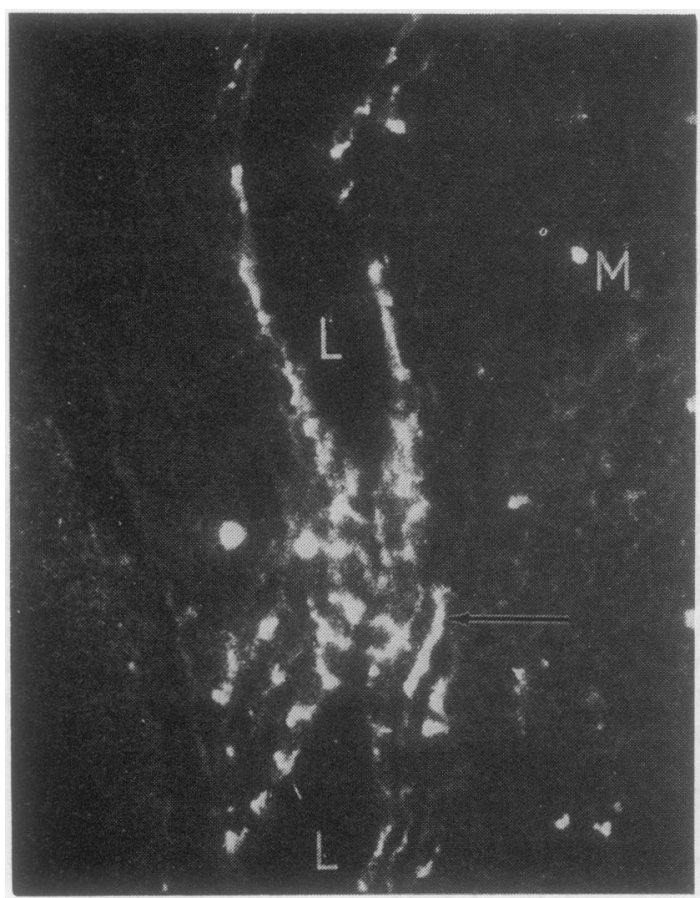

Fig. 1 Noradrenergic nerve fibres associated with a rat bronchial artery cut in tangential section. Most of the nerve fibres are non-varicose (arrow) ( $M=$ mast cell; $L=$ bronchial artery lumen). Fluorescence method $\times 120$. the blood vessel. When observed in tangential section, they form a seemingly continuous plexus around and along the artery, a pattern noted to continue along the branches of the bronchial artery (Fig. 1). The fluorescent bronchial arterial nerves appear as both varicose and non-varicose networks of fibres, but the dense varicose plexuses are observed more frequently (Figs 1 and 2). The loosely intermeshed non-varicose networks of fluorescent fibres wind spirally around the arterial wall in the adventitial layer (Fig. 1, arrow). The varicose adrenergic nerve plexuses of the bronchial arteries are randomly distributed in the adventitio-medial area. They often penetrate the media and appear in intimate relation to it (Fig. 3). The number of non-varicose fibres decreases sharply as sectioning proceeds deep into the pulmonary parenchyma. The non-varicose as well as the varicose adrenergic nerves of the bronchial artery contribute innervation to other components of the rat lung, including the bronchi, the pulmonary arteries, and the pulmonary veins.

The airways of the rat lung receive relatively few adrenergic nerve fibres. Most of those that

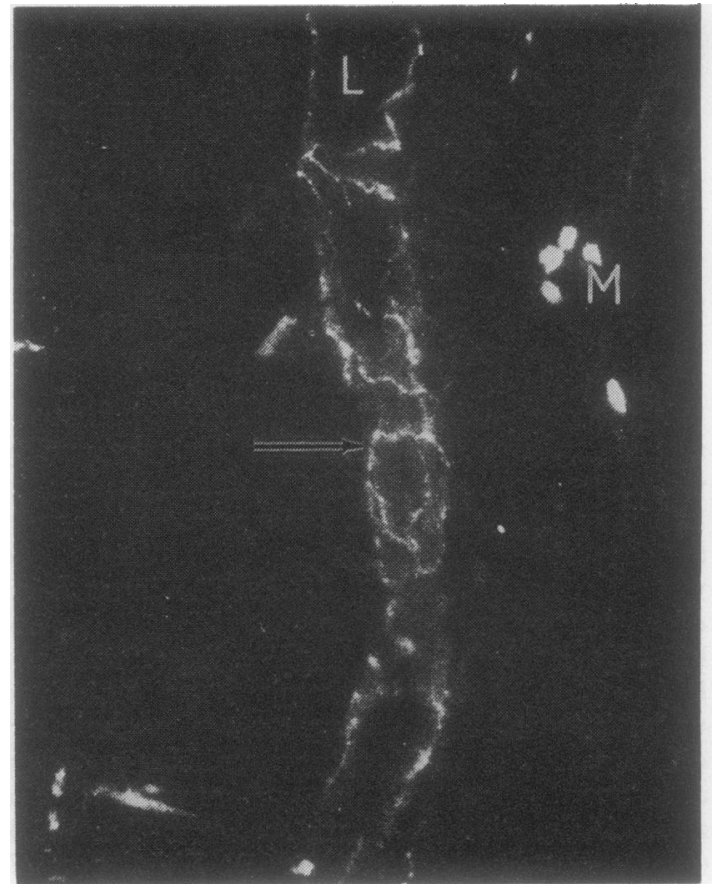

Fig. 2 Varicose noradrenergic nerves (arrow) associated with a rat bronchial artery cut in tangential and cross section ( $L=$ bronchial artery lumen; $M=$ mast cell). Fluorescence method $\times 120$. 
Fig. 3 A cross section of rat bronchial artery at the hilum. A heavily varicose plexus of noradrenergic nerves is located in the adventitio-medial area (arrow) and penetrates the media of the blood vessel ( $L=$ bronchial artery lumen). Fluorescence method $\times 220$.

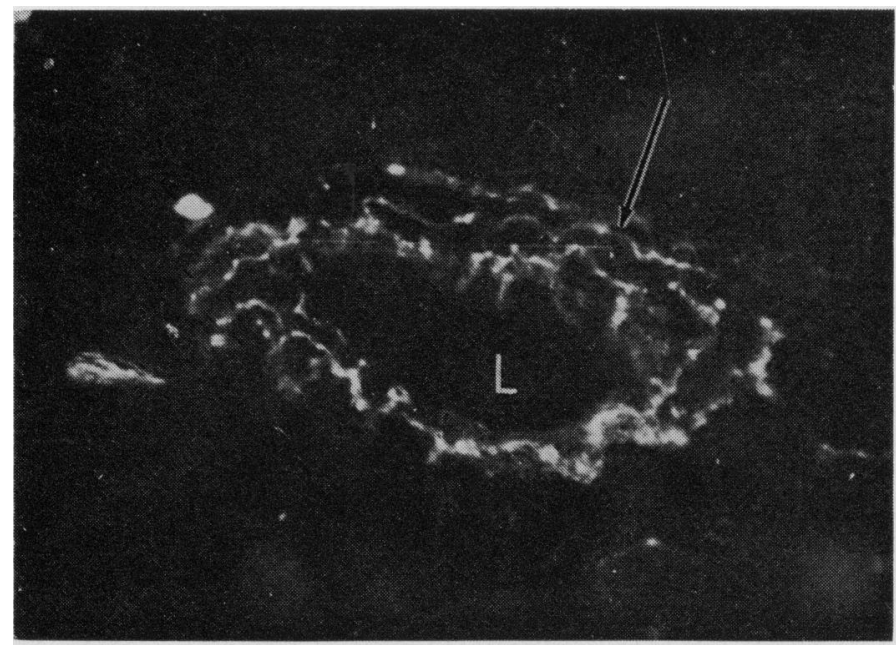

are present originate from the bronchial artery plexus. They pass as non-varicose fibres with the blood vessels between the bronchial or bronchiolar smooth muscle bands and extend through the submucosa, but no definite endings of these fluorescent fibres are apparent in the submucosal or mucosal areas. Although the small bronchioles usually follow this pattern, most segments demonstrating no fluorescent nerves in association with the smooth muscle, a small number of varicose nerves are found in association with bronchiolar smooth muscle at bifurcation points (Fig. 4).

Another structure of the rat lung which receives noradrenergic nerves is the pulmonary artery and its divisions. These fluorescent fibres also originate from the bronchial artery nerve plexus since in serial sections they can be traced from this source. They enter the pulmonary arterial wall as non-varicose fibres surrounding the vasa vasorum. In the media these non-varicose fibres are distributed to varicose plexuses within the smooth muscle layer (Fig. 5). These plexuses are more pronounced at branching points of the pulmonary artery, where there is an apparent increase in the number of fluorescent nerves.

The small pulmonary veins are also supplied with noradrenergic fibres. The larger veins, containing cardiac muscle, give no fluorescence reac-

Fig. 4 Varicose noradrenergic nerves in the smooth muscle (sm) layer of rat bronchial wall. Section is oblique through a bronchial bifurcation point $(L=$ bronchial lumen; $m=$ mucosal layer). Fluorescence method $\times 220$.

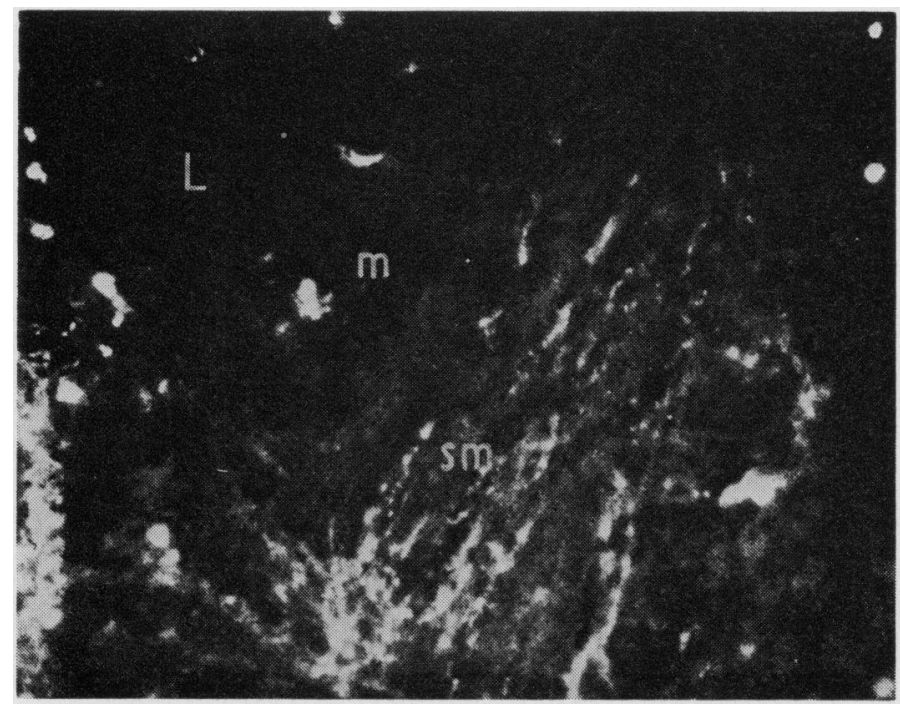




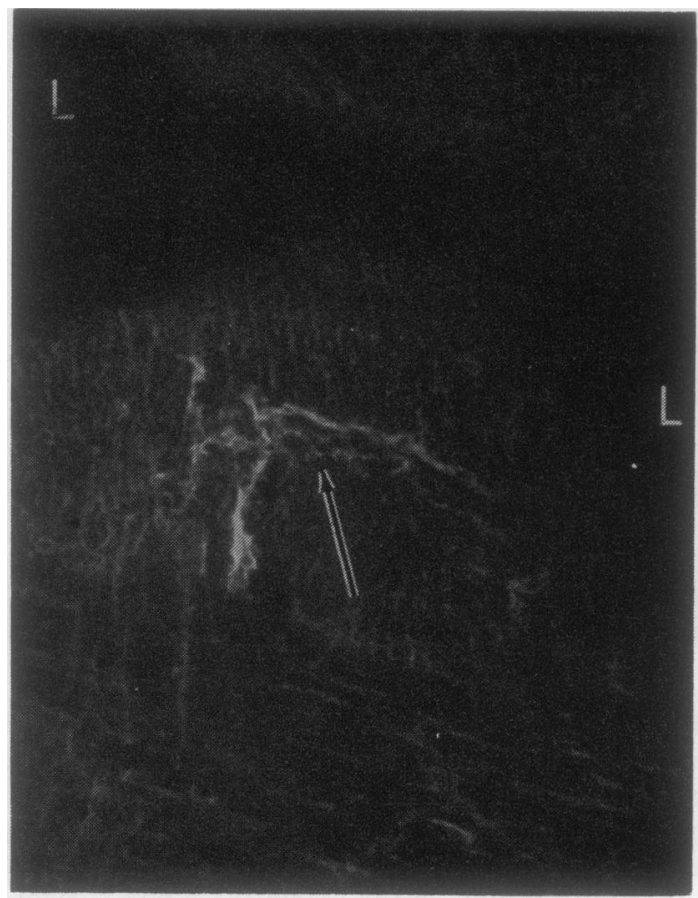

Fig. 5 Non-varicose noradrenergic nerves associated with a vas vasorum (arrow) in a cross section of pulmonary artery. These nerve fibres enter the arterial wall along with the vasa vasorum and give rise to varicose fibres deep in the medial layer $(L=$ pulmonary artery lumen). Fluorescence method $\times 120$.

tion for catecholamines. The noradrenergic nerve plexuses of the smaller veins are concentrated at the adventitio-medial junction in the rat. These plexuses extend longitudinally and circumferentially in the vein wall (Fig. 6) and are densely varicose. We have observed them along the divisions of the pulmonary vein up to the fifth branching. The larger branches of the rat pulmonary veins lack noradrenergic innervation.

\section{MONKEY}

In the Rhesus monkey, the major bronchial arteries are the main path of entrance of adrenergic nerves into the lung parenchyma. These fluorescent nerve plexuses are concentrated at the adventitio-medial junction and appear as a nonvaricose network (Fig. 7). A few divisions later, the bronchial arterial walls have a dense noradrenergic plexus, varicose in type and derived from the adventitial nerves. A longitudinal section through a third-generation bronchial artery at the hilum (Fig. 8) shows dense noradrenergic fibres penetrating the medial layer of the blood vessel.

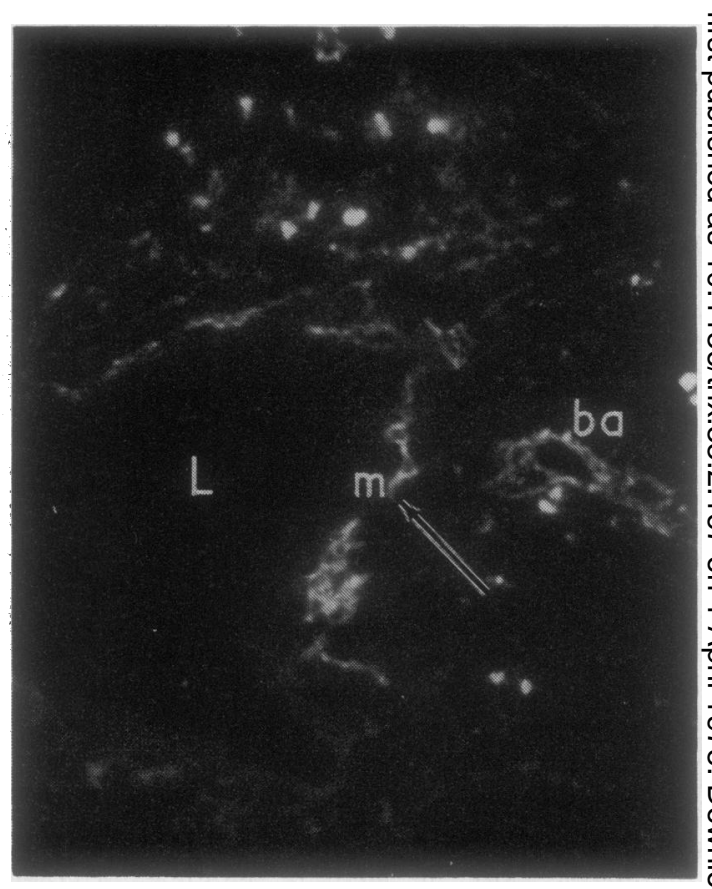

Fig. 6 Noradrenergic nerves associated with a pulmonary vein deep in the rat lung parenchyma. This dense nerve plexus is located at the adventitiomedial junction (arrow). A section of bronchial artery (ba) nearby also demonstrates adrenergic nerves ( $L=$ lumen; $m=$ media). Fluorescence method $\times 120$.

The nerves in the medial layer are highly varicose. $\stackrel{\nexists}{x}$ The distribution of the major branches of the bronchial arteries is apparently important in the dissemination of adrenergic nerves. Bronchialo arteries are found in the extrachondral connective tissue of the intrapulmonary bronchi and in bron-응 chiolar walls. Small branches of the bronchials artery are associated with the pulmonary blood을 vessels. In each case, nerve plexuses associated N with the bronchial arteries give rise to adrenergic nerves in the tissue supplied by the blood vessel.

The muscle layer of both bronchi and bronchioles in the monkey contains some associated fluorescent nerve fibres. In some instances theo bronchial arterial noradrenergic nerves can be $\bar{\Phi}$ seen in continuity with the fluorescent plexus on ${ }^{?}$ the bronchial muscle layer (Fig. 9). Deep in the lung, however, small bronchi and bronchioles do $\frac{\vec{P}}{\mathbb{D}}$ not demonstrate any continuity between the noradrenergic nerves of their muscle layers and $\mathbb{Q}$ those associated with the bronchial arteries. Theyo may have their origin in the adrenergic nerves $\Omega$ associated with larger bronchi travelling along응 


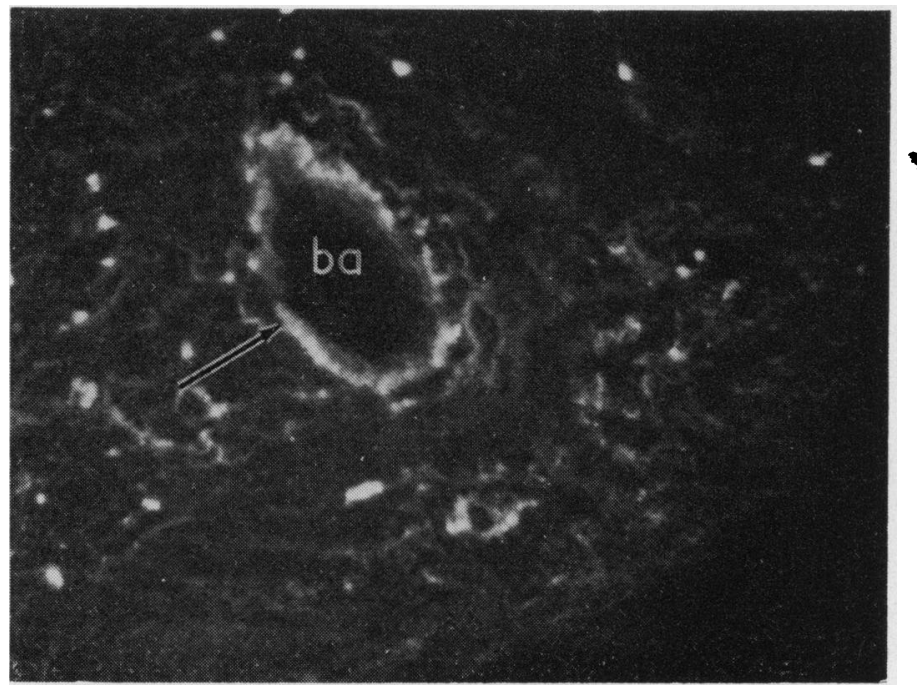

Fig. 7 Non-varicose noradrenergic nerves concentrated at the adventitiomedial junction of monkey bronchial artery (arrow) (ba=bronchial artery). Fluorescence method $\times 120$.

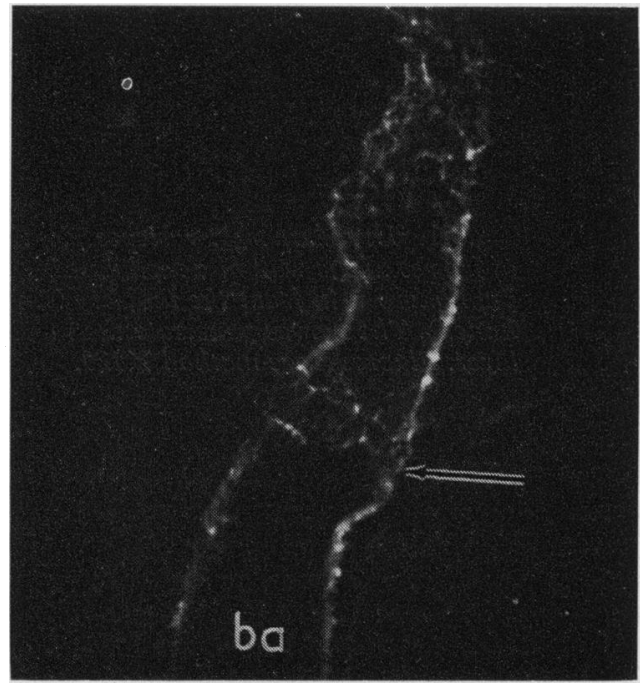

Fig. 8 Varicose noradrenergic nerve fibres (arrow) in the wall of a longitudinally sectioned monkey bronchial artery at the hilum (ba=bronchial artery). Fluorescence method $\times 120$.

the divisions of the bronchi and bronchioles themselves.

The pulmonary arteries of the monkey lung also are invested with noradrenergic innervation (Fig. 10). These nerves are located in the adventitio-medial junction. There is a high density of fluorescent nerve fibres at bifurcation points along the pulmonary artery, a pattern which is evident at all levels. The pulmonary artery nor-

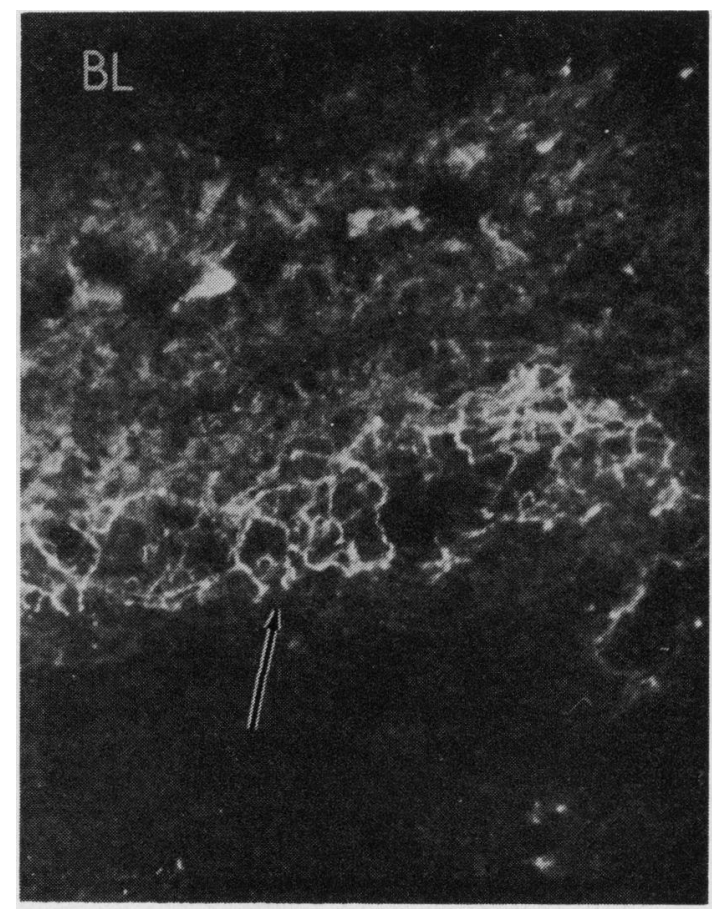

Fig. 9 Varicose plexus of noradrenergic nerve fibres (arrow) in relation to the muscular layer of a monkey bronchiole. This plexus is continuous with that of the bronchial artery $(B L=$ bronchial lumen). Fluorescence method $\times 120$.

adrenergic nerves are varicose but we have never seen them enter the medial layer. 


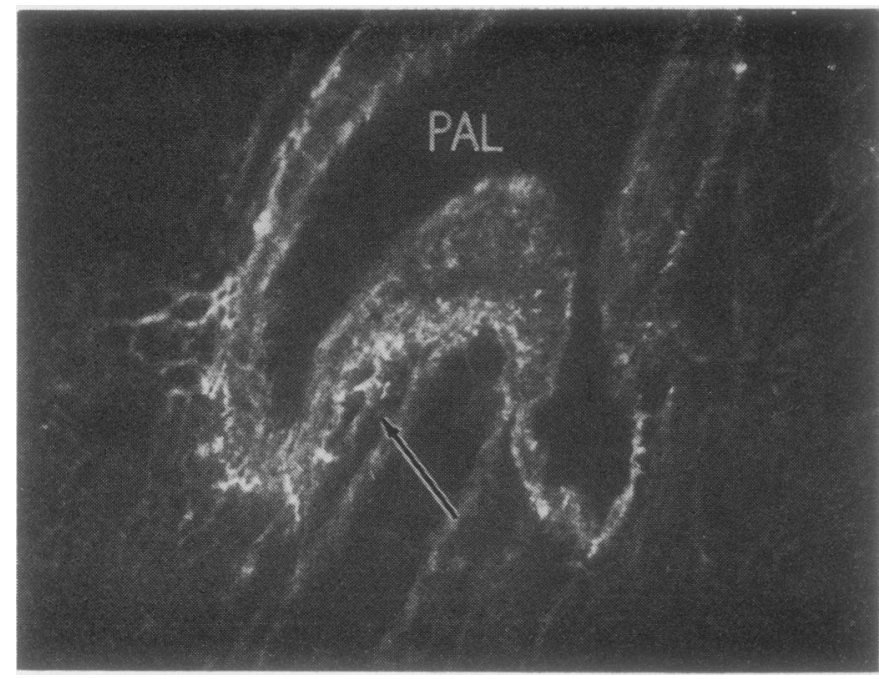

Fig. 10 Dense varicose and non-varicose noradrenergic nerves (arrow) at the adventitio-medial junction of a monkey medium-size pulmonary artery. Increased density of noradrenergic nerves is seen at a bifurcation point (arrow) (PAL= pulmonary artery lumen). Fluorescence method $\times 120$

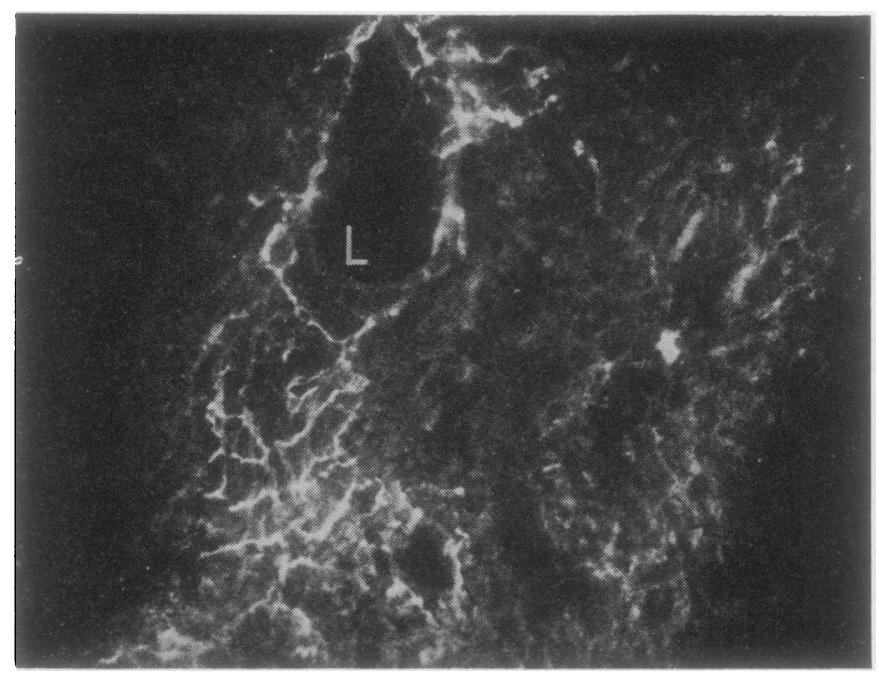

Fig. 11 Noradrenergic varicose plexus of nerves in the wall of a medium-size pulmonary vein from the monkey ( $L=$ lumen). The nerves penetrate the medial layer. Fluorescence method $\times 120$.

Noradrenergic innervation is also present in the pulmonary veins of the monkey. As in the rat, this innervation appears in the medium- and smalldiameter pulmonary veins. In contrast to the rat, the large pulmonary veins of the monkey also contain noradrenergic fibres, although these are somewhat less dense than those in the medium and small veins. The noradrenergic nerves of the medium and small pulmonary veins are varicose and penetrate both the adventitial and medial layers (Fig. 11).

\section{Discussion}

The presence of varicosities is a well-accepted indication of functional neuron terminals (Nor- $\widetilde{N}$ berg and Hamberger, 1964; Falck and Owman, 1965; Bennett, 1971). We therefore conclude that $\mathrm{N}$ the varicose fibres observed in the present studies in the medial layer of the rat and monkey bronchial artery wall are terminal. This finding is at variance with those of Čech and Doležel (1967) and Cech (1969), who stated that, as in the systemic vasculature, the terminals of the bronchial arterial innervation are concentrated in the adventitial layer. Ehinger (1964) demonstrated some terminals on smooth muscle cells of the arteries of the iris. Keatinge (1977) has found 2 differences in sensitivity to catecholamines between the inner and outer muscle layers of 
systemic arteries, the inner layer being the more sensitive. This higher sensitivity is thought to allow for response to the small amounts of noradrenaline which diffuse from the adventitial layer. Since our findings indicate more direct innervation of the medial smooth muscle layers in the bronchial arteries, one would predict more closely controlled physiological behaviour.

There are major differences in the general pattern of innervation of the rat lung and the monkey lung, as well as specific differences in the noradrenergic innervation between the two species. In both rat and monkey, the noradrenergic nerves enter the pulmonary parenchyma mainly in association with the bronchial arteries. In the rat, these noradrenergic nerves are distributed to the medial layer of the bronchial artery itself, even in its major divisions. In the monkey, however, noradrenergic nerves located in the adventitio-medial junction travel two to three generations along the intrapulmonary bronchial arteries before investing the medial smooth muscle with terminal varicose networks. In addition to these noradrenergic nerves, some bronchial arteries of the monkey have associated with them acetylcholinesterase-containing nerves arranged in bundles and plexuses (El-Bermani and Grant, 1975). Alternate sections subjected to different histochemical procedures indicate that the acetylcholinesterase-containing nerves are not coincident with the catecholamine-containing nerves (ElBermani and Grant, 1975). Thus, the bronchial artery of the monkey receives a dual innervation. We have not observed acetylcholinesterase-containing nerves in association with the bronchial arteries of the rat, despite positive reactions for the enzyme in other structures of the same sections. Thus, the autonomic innervation of the rat bronchial artery is exclusively noradrenergic. This is contrary to the finding of Cech (1969), who studied the monkey, rat, and cat and stated that bronchial arteries are innervated uniformly in all species.

In both the rat and the monkey, the noradrenergic nerves which enter the lung with the bronchial artery are distributed to the bronchi. The patterns by which these nerves extend to the airways, their density in the bronchial walls, and, probably most important, their distribution within the bronchi themselves are quite different. In the rat, medium and small bronchi receive noradrenergic nerves from the bronchial arteries. Once in the bronchial or bronchiolar wall these nerves pass through the smooth muscle bands without forming varicose terminal plexuses. They continue through the submucosa, extending towards the mucosal layer.
In the monkey however, the noradrenergic nerves enter the walls of the large (1-3 generation) bronchi in association with branches of the bronchial artery. Once in the bronchial wall, these noradrenergic nerves form a dense terminal plexus in the smooth muscle layer, especially at bronchial bifurcation points. Smaller bronchi and bronchioles also have a dense noradrenergic innervation in their smooth muscle layers. Since these nerves do not have continuity with those associated with the bronchial arteries, we presume that they originate from the adrenergic innervation of the larger bronchi. The sparsity of noradrenergic innervation of the bronchial wall has also been reported by Bennett and Malmfors (1970).

Differences exist in the noradrenergic innervation of the pulmonary arteries in the two species. In the rat, the noradrenergic nerves are associated mainly with the vasa vasorum, which penetrate the media of the pulmonary artery and can be traced back to bronchial arteries (El-Bermani et al., 1970). This finding differs from that of Cech (1969), who did not identify noradrenergic nerves in association with the rat pulmonary artery. Our findings are similar to those of Bennett (1971), who described adrenergic nerves associated with the vasa vasorum in the pulmonary artery of the fowl and confirmed their adrenergic origin by showing degeneration after treatment with 6-hydroxydopamine. In the monkey, the noradrenergic nerves are concentrated at the adventitio-medial junction of the pulmonary arteries and their divisions, a finding consistent with that of Cech (1969). The pulmonary veins of the rat and the monkey also show some differences in the pattern of their innervations. The noradrenergic innervation in the rat is restricted to the small pulmonary veins. In contrast, the noradrenergic nerves are distributed to all sizes of pulmonary vein in the monkey. The latter is similar to the pattern of pulmonary vein innervation described by Fillenz (1970) in the dog. In the rat we have observed that the pulmonary vein noradrenergic nerves are restricted to the adventitio-medial junction. In the monkey these nerves penetrate and form terminal plexuses in the medial layer. Cech (1969) could not demonstrate innervation in the small pulmonary veins of several species. The large pulmonary veins of the rat, which are devoid of noradrenergic nerves, contain a substantial number of cholinergic nerves and ganglia (El-Bermani and Grant, 1975).

A striking finding of the present studies is the prevalence of noradrenergic nerve terminals in association with the vasa vasorum. The response 
of the pulmonary vasculature to various humoral substances may thus be regulated to some extent by variations in their delivery to the vascular wall.

This work was supported by grant No. HL20548 from the National Heart and Lung Institute.

I express my appreciation for the help and criticism of Dr. Eunice I. Bloomquist who read the manuscript.

\section{References}

Altenähr, E. (1965). Untersuchungen über die Feinstruktur der vegetativen Innervation der Rattenlunge. Zeitschrift für MikroskopischAnatomische Forschung, 72, 439-518.

Bennett, T. (1971). The adrenergic innervation of the pulmonary vasculature, the lung and the thoracic aorta, and on the presence of aortic bodies in the domestic fowl (Gallus gallus domesticus L.). Zeitschrift für Zellforschung und Mikroskopische Anatomie, 114, 117-134.

Bennett, T., and Malmfors, T. (1970). The adrenergic nervous system of the domestic fowl (Gallus domesticus L.). Zeitschrift für Zellforschung und Mikroskopische Anatomie, 106, 22-30.

Cech, S. (1969). Adrenergic innervation of blood vessel in the lung of some mammals. Acta Anatomica, 74, 169-182.

Čech, S., and Doležel, S. (1967). Monoaminergic innervation of the pulmonary vessels in various laboratory animals (rat, rabbit, cat). Experimentia, 23, 113-115.

Ehinger, B. (1964). Adrenergic nerves to the eye and its adnexa in rabbit and guinea pig. Acta Universitatis Lundensis, Section 2. Medica, Mathematica, Scientiae Rerum Naturalium, 20, 1-23.

Ehinger, B., Falck, B., and Sporrong, B. (1967). Adrenergic fibres to the heart and to peripheral vessels. Bibliotheca Anatomica, 8, 35-45.

El-Bermani, Al-W., and Grant, M. (1975). Acetylcholinesterase-positive nerves of the rhesus monkey bronchial tree. Thorax, 30, 162-170.

El-Bermani, Al-W., McNary, W. F., and Bradley, D. E. (1970). The distribution of acetylcholinesterase and catecholamine containing nerves in the rat lung. Anatomical Record, 167, 205-207.

Falck, B. (1962). Observations on the possibilities of the cellular localization of monoamines by a fluorescence method. Acta Physiologica Scandinavica, 56, Supplement No. 197.

Falck, B., and Owman, C. (1965). A detailed methodological description of the fluorescence method for the cellular demonstration of biogenic $\stackrel{\overrightarrow{\vec{D}}}{\vec{O}}$ monoamines. Acta Universitatis Lundensis, Section 0 2. Medica, Mathematica, Scientiae Rerum Naturalium, 7, 1-23.

Fillenz, M. (1967). Innervation of blood vessels of $\vec{\nabla}$ lung and spleen. Bibliotheca A natomica, 8, 56-59.

Fillenz, M. (1970). Innervation of pulmonary and $\nRightarrow$ bronchial blood vessels of the dog. Journal of $\vec{\circ}$ Anatomy, 106, 449-461.

Fisher, A. W. F. (1965). The intrinsic innervation of the pulmonary vessels. Acta Anatomica, 60, 481496.

Fuxe, K., and Sedvall, G. (1965). The distribution of $\underset{\omega}{\dot{\omega}}$ adrenergic nerve fibers to the blood vessels in skeletal muscle. Acta Physiologica Scandinavica, ? 64, 75-86.

Hayek, H. von (1960). The Human Lung, p. 315. Hafner Publishing Company, New York.

Honjin, R. (1956). On the nerve supply of the lung of the mouse, with special reference to the structure $\frac{D}{2}$ of the peripheral vegetative nervous system. Journal of Comparative Neurology, 105, 587-625.

Keatinge, W. R. (1977). Differences in electrical and mechanical behaviour of inner and outer smooth muscle of arteries. Proceedings of the International Union of Physiological Sciences, 12, 335.

Larsell, O. (1921). Nerve terminations in the lung of the rabbit. Journal of Comparative Neurology, 33, 105-131.

Larsell, O. (1922). The ganglia, plexuses, and nerveterminations of the mammalian lung and pleura $\overrightarrow{\bar{O}}$ pulmonalis. Journal of Comparative Neurology, $\exists$ 35, 97-132.

Lockett, M. (1957). The transmitter released by stimulation of the bronchial sympathetic nerves in the cat. British Journal of Pharmacology, 12, 8696.

Norberg, K. A., and Hamberger, B. (1964). The sympathetic adrenergic neuron. Some characteristics revealed by histochemical studies on the intra-neuronal distribution of the transmitter. Acta Physiologica Scandinavica, 63, Supplement No. 238.

Zussman, W. V. (1966). Fluorescent localization of catecholamine stores in the rat lung. Anatomical Record, 156, 19-23.

Requests for reprints to: Dr. Al-W. I. El-Bermani, Department of Anatomy, Tufts University School of $\stackrel{\varrho}{\subset}$ Medicine, 136 Harrison Avenue. Boston, Massachusetts 02111 . USA. 